\title{
New insights into novel Escherichia coli colistin-resistant strains isolated from Argentina
}

\author{
Patricia Maturana ${ }^{1,4} \cdot$ Melina Martinez $^{2,4} \cdot$ Diego Faccone $^{3,4} \cdot$ Liliana Semorile $^{2} \cdot$ Paulo C. Maffia ${ }^{2,4} \cdot$ Axel Hollmann $^{1,2,4}$
}

Received: 1 July 2019 / Revised: 16 April 2020 / Accepted: 28 April 2020 / Published online: 11 May 2020

(c) European Biophysical Societies' Association 2020

\begin{abstract}
Colistin is a polymyxin antibiotic (polymyxin E) that has in recent years re-emerged as an option for treatment of multidrugresistant bacteria. Recently, the re-introduction of colistin resulted in the appearance of colistin-resistant bacteria, which is usually caused by LPS modifications. The fact that this modification is mediated by a plasmid carrying the mcr-1 gene, implies a horizontal transfer of colistin resistance. In Argentina, the National Reference Laboratory in Antimicrobial Resistance (NRLAR), has recently screened several bacteria for the MCR-1 plasmid, detecting nine Escherichia coli isolates carrying the plasmid with the mcr-1 gene, among others. In this context, we proposed to assess the effect of surface charge modifications induced by the plasmid MCR-1 and its impact on the resulting colistin resistance in two clinical isolates of colistin-resistant $E$. coli. Using zeta potential assays, we confirmed the reduction of negative charge exposure on clinical isolates compared to the reference strain of $E$. coli. In addition, through permeabilization assays, we were able to correlate this reduction in charge exposure with the extent of damage to the bacterial membrane. The fact that this surface charge modification through substitution of lipid A is plasmid encoded, represents an important concern for future antimicrobial peptide drug development.
\end{abstract}

Keywords Colistin $\cdot$ Resistance $\cdot$ Membrane permeability $\cdot$ Potential zeta

\section{Introduction}

Patricia Maturana and Melina Martinez equal contribution.

Electronic supplementary material The online version of this article (https://doi.org/10.1007/s00249-020-01436-x) contains supplementary material, which is available to authorized users.

Axel Hollmann

ahollmann@gmail.com

1 Laboratorio de Compuestos Bioactivos, Centro de Investigaciones en Biofísica Aplicada y Alimentos (CIBAAL), CONICET, Universidad Nacional de Santiago del Estero, RN 9, Km 1125, 4206, Santiago del Estero, Argentina

2 Laboratorio de Microbiología Molecular, Instituto de Microbiología Básica y Aplicada, Universidad Nacional de Quilmes, Roque Saenz Peña 352, B1876BXD Bernal, Argentina

3 Servicio Antimicrobianos, Instituto Nacional de Enfermedades Infecciosas-ANLIS “Dr. Carlos G. Malbrán”, Av. Velez Sarsfield 563, C1282AFF Buenos Aires, Argentina

4 Consejo Nacional de Investigaciones Científicas y Técnicas (CONICET), Buenos Aires, Argentina
Colistin, a polymyxin antibiotic (polymyxin E), was first discovered in the 1940s but was not clinically used until the late 1950s. However, nephrotoxicity and neurotoxicity issues caused by this drug and the emergence of other antibiotics (e.g., aminoglycosides) that were less toxic, deterred physicians from using colistin. In this context, between the 1970s and 1990s, colistin was not often used (Li et al. 2006). Nevertheless, the lack of treatment options for multidrug-resistant (MDR) bacteria has led to the re-emergence of colistin as an antimicrobial therapy in recent years (Lim et al. 2010).

The antibacterial activity of polymyxins relies on a detergent-like effect, via a two-step mechanism. This comprises initial binding via electrostatic interactions between the polycationic ring of colistin to cell envelope components, specifically the lipid A portion of the lipopolysaccharide (LPS) that form the outer membrane of Gram-negative bacteria (Magiorakos et al. 2012; Liu et al. 2016; Poirel et al. 2017), causing the displacing, in a competitive fashion, of calcium $\left(\mathrm{Ca}^{2+}\right)$ and magnesium $\left(\mathrm{Mg}^{2+}\right)$ ions from the phosphate groups of LPS, that act as membrane stabilizers 
(Bialvaei and Samadi Kafil 2015). Consequently, the outer membrane is destabilized, increasing its permeability and leading to leakage of the cytoplasmic contents with subsequent lysis and bactericidal activity (Esposito et al. 2017).

The re-introduction of colistin in recent years resulted in the appearance of colistin-resistant bacteria, which is usually caused by LPS modifications (Liu et al. 2016). In most resistant strains, 4-amino-4-deoxy-L-arabinose (L-Ara4N), phosphoethanolamine $(\mathrm{PEtN})$ or galactosamine moieties are enzymatically added to the lipid A on the LPS core (Magiorakos et al. 2012; Liu et al. 2016; Poirel et al. 2017). The plasmid-mediated mcr-1 gene, responsible for horizontal transfer of colistin resistance, was first described for Escherichia coli and Klebsiella pneumoniae isolates recovered in China between 2011 and 2014 (Liu et al. 2016). The encoded MCR-1 protein is a member of the phosphoethanolamine transferase enzyme family, as its acquisition results in the addition of phosphoethanolamine to lipid A, and consequently in a more cationic LPS that impairs or diminishes colistin attachment (Poirel et al. 2017).

In Argentina, the National Reference Laboratory in Antimicrobial Resistance (NRLAR), from 2008 to January 2016 has screened several bacteria for the MCR-1 plasmid. In particular, the mcr- 1 gene was detected in nine $E$. coli isolates (Rapoport et al. 2016).

In this study, we aimed to evaluate the effect of surface charge modifications induced by the plasmid MCR-1 on the resulting colistin resistance, by zeta potential and membrane permeabilization assays in two clinical isolates of $E$. coli carrying the MCR-1 plasmid.

\section{Materials and methods}

\section{Bacterial strains and growth conditions}

E. coli ATCC25922, and the two E. coli clinical isolates M15049 and M15224 resistant to colistin but carrying the $m c r-1$ gene used in this work were previously characterized (Rapoport et al. 2016). To carry out all the tests, strains were grown in Mueller Hinton Broth (MHB, Britania, Buenos Aires, Argentina) at $37{ }^{\circ} \mathrm{C}$. Minimal inhibitory concentration (MIC) was determined by standard microdilution assays according to CLSI recommendations (Clinical and Laboratory Standards Institute 2015), using MH media in the presence and absence of $80 \mu \mathrm{g} / \mathrm{ml}$ of EDTA.

\section{Zeta potential}

Zeta potential, determined by dynamic light scattering (DLS), was performed at $25^{\circ} \mathrm{C}$ on a Horiba SZ-100 nanoparticle analyzer (Horiba, Kyoto, Japan). Each overnight culture of bacteria in MHB (with and without $80 \mu \mathrm{g} / \mathrm{ml}$ of
EDTA) was washed twice in saline solution $(\mathrm{NaCl} 0.89 \%$ $\mathrm{w} / \mathrm{v}$ ) and diluted to reach a bacterial concentration of $3 \times 10^{7}$ Colony Forming Units (CFU)/mL to acquire enough count rates. Zeta potential was determined from the mean of 10 measurements (500 runs each). The complete experiment was carried out in triplicate for each sample, using independently grown cultures. The $\mathrm{pH}$ of the final solution of bacteria was 6.5. Due to our experimental conditions (i.e. work in ionic media and measuring particles of relatively high diameter $>200 \mathrm{~nm}$ ), zeta potential was calculated from cell mobility by using the Smoluchowski approximation, and Henry's function was set at 1.5 (Lowry et al. 2016).

zeta potential $=\frac{3 \mu E \eta}{2 \varepsilon f(\kappa r)}$,

where $\mu E$ is the electrophoretic mobility; $\eta$ is the dispersion medium viscosity; $\varepsilon$ is the dispersion medium dielectric constant and $f(\kappa r)$ is Henry's function. Zeta potential changes induced by colistin addition after bacteria were incubated for $1 \mathrm{~h}$ with $4 \mu \mathrm{g} / \mathrm{ml}$ of colistin, were also determined.

\section{Outer membrane (OM) permeabilization}

OM permeabilization activity was determined by using the $1-\mathrm{N}$-phenylnaphthylamine (NPN) fluorescent assay, as previously described (Dong et al. 2014). Measurements were carried out on a Varian Cary Eclipse fluorescence spectrophotometer (Mulgrave, Australia), with excitation at $350 \mathrm{~nm}$ and emission at $420 \mathrm{~nm}$. Briefly, an overnight culture of each strain was inoculated in fresh MHB media and incubated at $37{ }^{\circ} \mathrm{C}$ under agitation until reaching an OD $600 \mathrm{~nm}$ of 0.1 . Cells were harvested by centrifugation at $5000 \times g$ for $5 \mathrm{~min}$, washed twice in PBS, and later $600 \mu \mathrm{l}$ of cell suspension was mixed with an aliquot of NPN to a final probe concentration of $12 \mu \mathrm{M}$. Subsequently, cell suspension was added to a $0.5 \mathrm{~cm}$ quartz cuvette and fluorescence was recorded over $1 \mathrm{~min}$, flowed by the addition of $4 \mu \mathrm{g} / \mathrm{ml}$ colistin (time 0 ), mixed by pipetting up and down, and fluorescence was continuously recorded over time until no further increase was detectable. The sigmoidal model used to characterize the kinetics uptake of NPN is described by following equation (Batzli and Love 2015):

$F=F_{\text {max }}+\frac{F_{0}-F_{\text {max }}}{1+\mathrm{e}^{k\left(t-t_{50}\right)}}$,

where $F_{0}$ represents the fluorescence baseline before NPN addition and $F_{\text {max }}$ the maximum value achieved after colistin addition, $\mathrm{F}$ is fluorescence measured. The time constant, $t_{50}$, is the time to toggle from the initial to half the fluorescence of the final state of uptake, and $k$ (units of time ${ }^{-1}$ ) is a kinetic time constant related to the inverse of the time to complete the transition beyond the lag time. The lag time is related 
to the sigmoidal time constants by the following equation (Batzli and Love 2015):

$t_{\text {lag }}=t_{50}-\frac{2}{k}$.

The complete experiment was carried out in triplicate for each sample, using independently grown cultures.

\section{Fluorescence microscopy}

To evaluate cell viability, a staining procedure was performed with solutions of thiazole orange (TO) and propidium iodide (PI) to differentiate the living and membrane damaged bacteria. Bacterial suspensions were prepared following manufacturer instruction in the $\mathrm{BD}^{\mathrm{TM}}$ Cell Viability Kit (BD Biosciences, USA). Briefly, an overnight culture of each strain was inoculated in fresh MHB media and incubated at $37{ }^{\circ} \mathrm{C}$ with agitation until reaching an OD $600 \mathrm{~nm}$ of 0.1. Afterward, both probes were added in a final concentration of $420 \mathrm{nmol} / 1$ for TO and $43 \mu \mathrm{mol} / \mathrm{l}$ for PI and incubated for $15 \mathrm{~min}$ with gentle agitation protected from light. Samples were imaged using an Olympus Inverted Fluorescent microscope CKX 41 (Olympus, Japan) coupled with a digital camera Olympus QColor3-RTV-R (Olympus).

\section{Data analysis}

Fitting of the equations mentioned in this work to the experimental data was done for non-linear regression using GraphPad Prism 5. Error bars on data presentation represent the standard error of mean (SEM).

\section{Results and discussion}

As was recently described, the clinical isolates M15049 and M15224 harboring the mcr-1 gene (Rapoport et al. 2016) displayed a colistin-resistant phenotype with a MIC of 8 and $16 \mu \mathrm{g} / \mathrm{ml}$, respectively. On the other hand, the MIC value described for the non-resistant ATCC 25922 bacterial strain is $0.5-2 \mu \mathrm{g} / \mathrm{ml}$ (Turlej-Rogacka et al. 2018). It was previously pointed out that the replacement of lipid A with the PEtN-4'-lipid A, mediated by MCR-1, reduced the negative membrane charge of all colistin-resistant E. coli (Fig. 1). Furthermore, the possibility of using zeta potential as a diagnostic tool for colistin-resistant strains has been recently postulated (Esposito et al. 2017). In this context, to corroborate this statement on new Argentinian colistin-resistant E. coli strains, the zeta potential of these strains and the colistinsensitive ATCC reference strain was characterized. As can

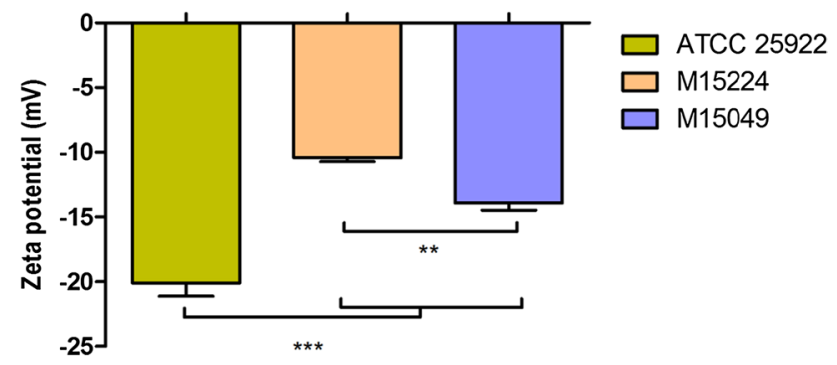

Fig. 2 Zeta potential values of bacteria. Each point represents the averages of three independent measurements. Error bars indicate standard deviations of means. All assays were carried out at $20{ }^{\circ} \mathrm{C}$. Statistical comparison using one-way ANOVA followed by a Turkey's post-test for multiple comparisons; $* * p<0.001$, $* * * p<0.0001$. $N=3$
A

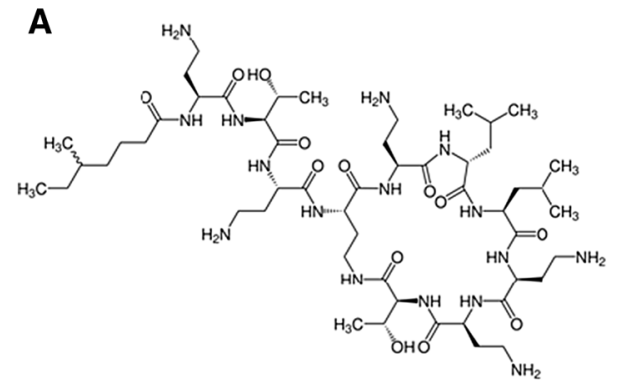

B

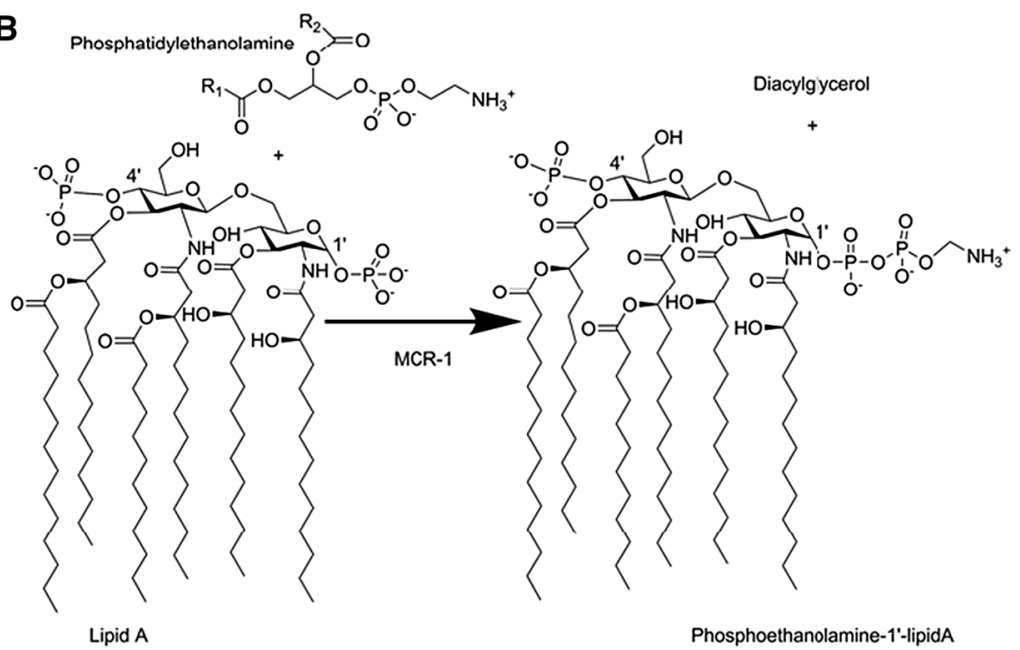

Fig. 1 a Structure of colistin and $\mathbf{b}$ the phosphoethanolamine transfer reaction catalysed by MCR-1 in E. coli lipid A (Hinchliffe et al. 2017) 
be seen in Fig. 2, both clinical isolates exhibited a significant (control vs. M15224 $p$ value $=0.000034$; control vs. M15049 $p$ value $=0.0005)$ reduction of zeta potential. Interestingly, the isolate M15224 with a higher MIC value toward colistin (Rapoport et al. 2016), showed less negative potential, reinforcing the hypothesis of the relationship between the zeta potential and resulting resistance. As was pointed out above, this reduction of zeta potential could be ascribed to the addition of PEtN at the 4' position of lipid A. Furthermore, the match on zeta potential reduction and MIC values found, allowed us to hypothesize that this difference in the zeta potential could be related with the degree of substitution of lipid A.

To get an insight into the effect of zeta potential changes, through lipid A substitution on bacteria and colistin interactions, we obtained zeta potential values of the reference susceptible strain and both resistant isolates after bacteria had been incubated for $1 \mathrm{~h}$ with $4 \mu \mathrm{g} / \mathrm{ml}$ of colistin. Interestingly, only the reference strain showed a significant zeta potential change ( $p$ value $=0.0268$ ), becoming less negatively charged after colistin addition (Fig. 3). As was pointed out above, colistin is a positively charged polypeptide with four positive charges at the $\mathrm{pH}$ tested (Shah et al. 2014). In this context, we could hypothesize that attachment of colistin to the bacterial outer membrane, through lipid A, should induce a reduction in zeta potential value. However, other factors related to the interaction of colistin with susceptible bacteria, such as lipid disorganization, could also explain this reduction in zeta potential and should not be ruled out. The fact that no changes were observed in both resistant strains could imply an insufficient interaction of colistin with the bacterial surface, or a reduction in the capacity of their interactions at the bacterial surface to induce a noticeable change in the zeta potential.

As was previously pointed out, masking the negatively charged phosphates of lipid A could induce a reduction in

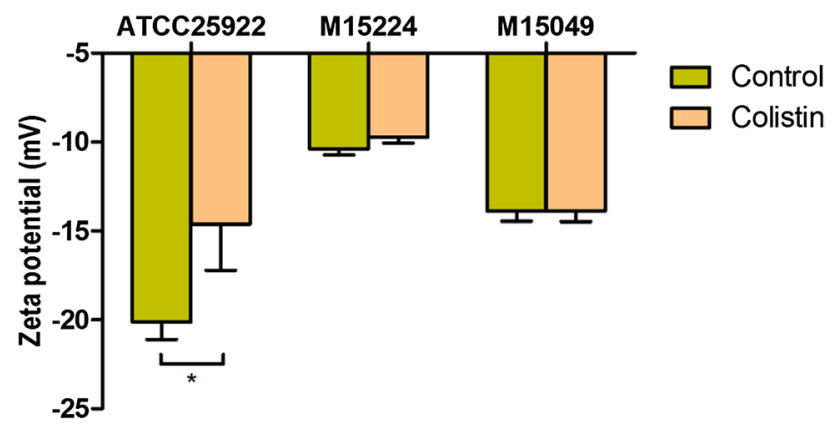

Fig. 3 Zeta potential values of bacteria incubated with $4 \mu \mathrm{g} / \mathrm{ml}$ of colistin. Each point represents the averages of three independent measurements. Error bars indicate standard deviations of means. All assays were carried out at $25{ }^{\circ} \mathrm{C}$. ${ }^{*} p<0.5$ unpaired $t$ tests between bacteria with and without colistin colistin affinity (Esposito et al. 2017), which is the initial electrostatic interaction that temporarily stabilizes the complex and brings the N-terminal fatty acyl chain of the polymyxin molecule into proximity with the outer membrane (Velkov et al. 2010). Therefore, inhibition at that initial stage could explain the ability of the bacteria to become resistant. It has been previously demonstrated that the phosphoethanolamine transferase enzyme family requires zinc for MCR activity (Hinchliffe et al. 2017), and regarding this, the removal of zinc through inclusion of the chelator EDTA reverted colistin-resistant $E$. coli isolates to a susceptible phenotype (Esposito et al. 2017; Hinchliffe et al. 2017). In this context, zeta potential and MIC values of two resistant isolates and the reference strain used in this study were determined in the presence of EDTA, showing in this case a reduction of MIC on both resistant bacteria to a similar MIC value to susceptible reference strain $(1 \mu \mathrm{g} / \mathrm{ml})$. Interestingly, this behaviour was concomitant with a decrease in zeta potential values of $6.2 \pm 0.7 \mathrm{mV}$ for clinical isolate M15224 and $5.1 \pm 0.5 \mathrm{mV}$ for M15049, reaching negative values comparable to the reference strain. Beside EDTA chelator activity that could act non-specifically affecting other bacterial processes, the fact that non-significant changes were observed in the susceptible reference strain (neither in MIC nor in zeta potential), reinforces the relationship between zeta potential values and colistin resistance.

Considering that both resistant strains showed no changes in zeta potential after colistin incubation, and taking into account that the colistin concentration used is lower than MIC values for both isolates, we aimed to evaluate if this sub MIC concentration is still capable of inducing some effects on the membrane permeabilization. As was previously published, the mode of action of colistin is related to its ability to disrupt the bacterial outer membrane (Falagas et al. 2005). In this context, we evaluated the ability of colistin to permeabilize the outer membrane of three $E$. coli strains using the NPN uptake assay. NPN is a small hydrophobic molecule that is excluded by intact bacterial outer membranes, but exhibits increased fluorescence after partitioning into disrupted outer membranes (Dong et al. 2014). Thus, an increase in fluorescence intensity in the presence of any agent indicates a disrupted or permeabilized bacterial outer membrane.

As shown in Fig. 4, the addition of colistin promoted NPN uptake across the outer membrane of three tested bacteria, confirming its ability to disrupt the outer bacterial membrane. As expected, E. coli ATCC 25922 showed the highest uptake value. Regarding the clinical isolates, M15224 exhibited a slightly lower uptake value in comparison with the isolate M15049. When uptake kinetics was analyzed, E. coli ATCC 25922 showed the fastest permeabilization behaviour (i.e. lower $t_{50}$ ), and this value was twofold lower than isolate M15049 and threefold lower than M15224 

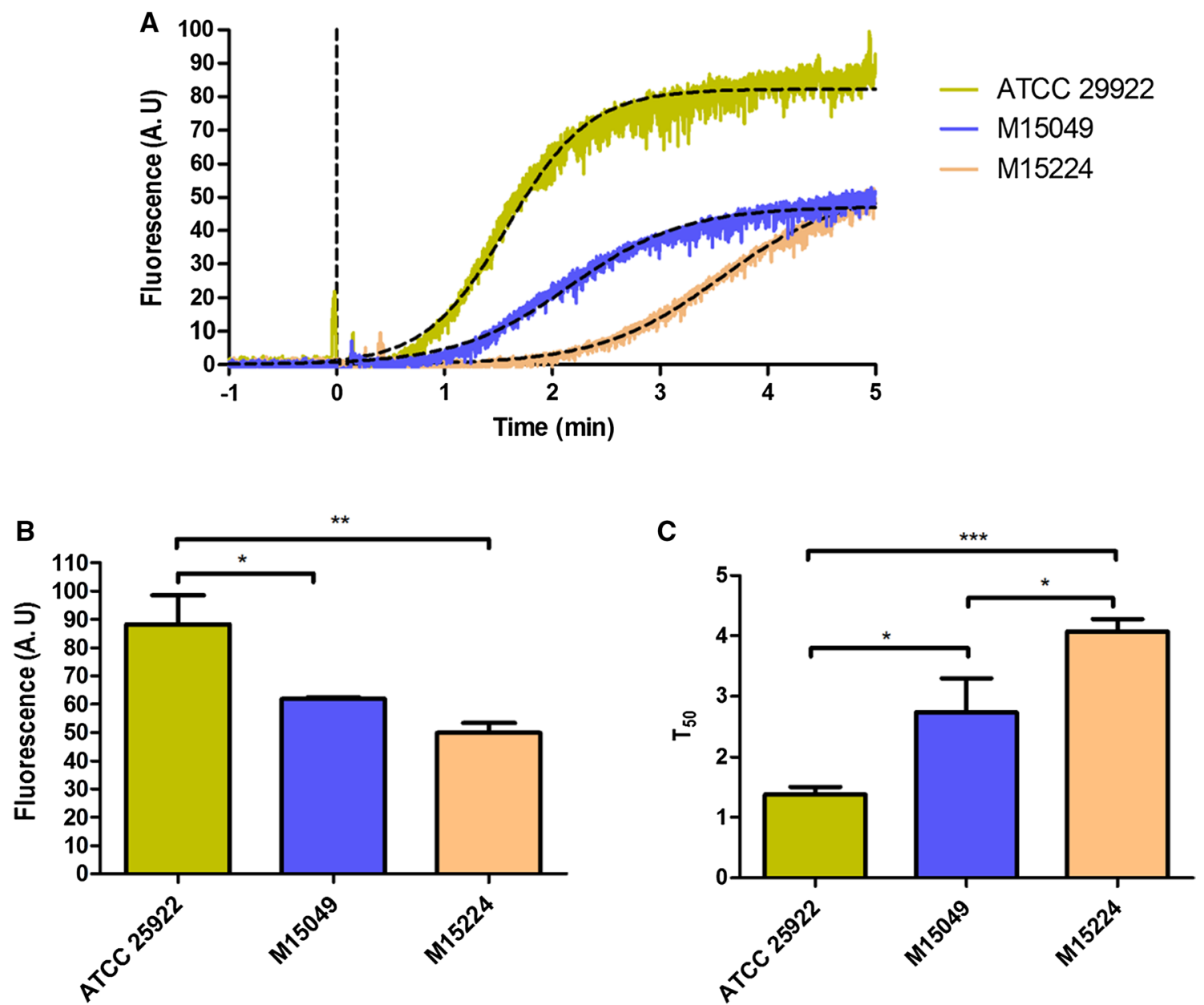

Fig. 4 Outer membrane permeabilization. Bacterial cells were incubated with NPN in the presence of $4 \mu \mathrm{g} / \mathrm{ml}$ of colistin. a NPN uptake kinetics measured by an increase in fluorescence intensity after probe partition into the hydrophobic core of the outer membrane, time $=0$ correspond with colistin addition. Dashed lines represent the fitting

(Fig. 4c). In this case, beside isolates M15049 and M15224 showing similar permeabilization rates, the first one exhibited significantly faster kinetics ( $p$ value $=0.0153$ ), in good agreement with MIC data. Also, $t_{\text {lag }}$ times for both clinical isolates where much higher (1.38 min for M15049 and 2.32 min for M15224) than for the sensitive ATCC strain $(0.61 \mathrm{~min})$. The fact that both clinical isolates show permeabilization or disruption of the outer membrane while no changes in zeta potential were observed implying that even small amounts of colistin attached to the membrane are enough to induce an effect. However, no inhibitory effect was observed at this colistin concentration, which implies that bacteria were able to overcome this partial disruption of the membrane. In order to evaluate this issue, a live/dead fluorescence assay was employed. As shown in Fig. 5, reference strains exhibited significant membrane damage (i.e. red stained bacteria) after $1 \mathrm{~h}$ of incubation with colistin,

of measured data with Eq. 2, b NPN uptake after 20 min. $\mathbf{c}$ Time to reach $50 \%\left(t_{50}\right)$ of NPN uptake. Data presented as mean \pm standard deviations of means. Statistical comparison using one-way ANOVA followed by a Turkey's post-test for multiple comparisons; ${ }^{*} p<0.05$, $* * p<0.001, * * * p<0.0001 . N=3$

whereas in both clinical isolates, most bacteria remained viable (i.e. green bacteria). Confirming that partial permeabilization of the outer membrane is not enough to kill bacteria, in good agreement with MIC data. When both clinical isolates were compared, although the clinical isolate M15049 showed mostly viable bacteria, some membrane damaged bacteria appeared, compared to clinical isolate M15244, in agreement with MIC and NPN uptake assays. It was previously pointed out that for many antimicrobial drugs, including antimicrobial peptides, rapid kinetics of bacterial killing can reduce the potential for the development of resistance compared to the slower kinetics observed with standard antimicrobial agents (Deslouches et al. 2013). In this context, the significantly slower kinetics, as well as higher $t_{\text {lag }}$ times of permeabilization observed in both clinical isolates, could allow the bacteria to adapt and become resistant. 

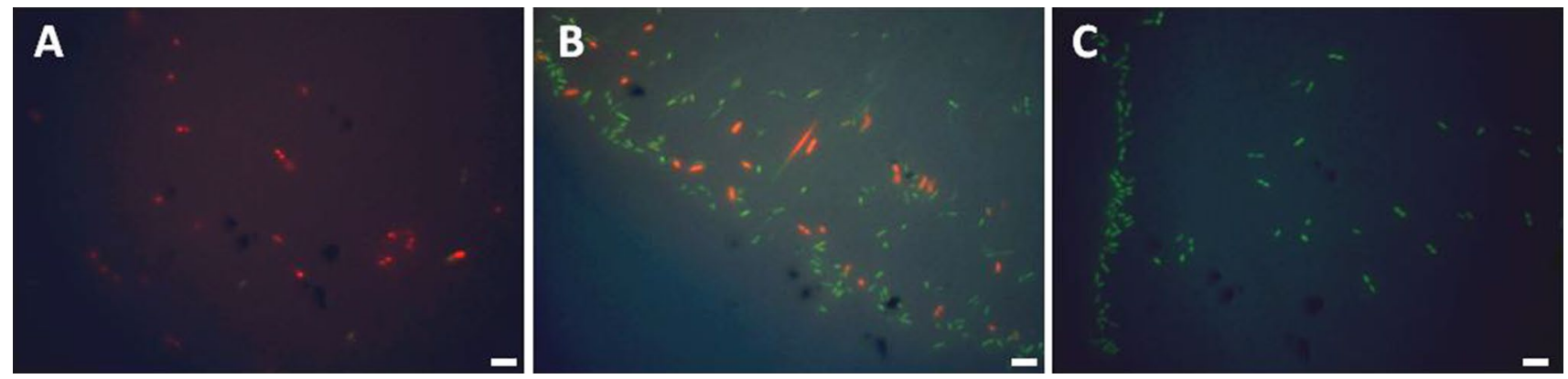

Fig. 5 Live/dead staining of $E$. coli after 1 h. of incubation with colistin. a ATCC 25955, b M15049 and c M15224. Scale bar $=5 \mu M$

The fact that the zeta potentials match with the peptide permeabilization and MIC results on both clinical isolates could suggest (1) a partial substitution of lipid A that reduces the possible colistin site of attachment, but leaves some nonmodified moieties where colistin could still attach but to a lower extent, or (2) a reduction of zeta potential, that reduces the electrostatic force that drives the first step in colistin mechanism of action. Also, a combination of both mechanisms should be considered, resulting in weaker interaction of colistin with the bacterial surface insufficient to induce lethal damage to the bacteria.

Besides lipid A being the specific target of colistin, the reduction of zeta potential by the MCR-1 protein could also have important consequences in other antimicrobial compounds, where electrostatic interactions drive the first steps of membrane attachment, as with many cationic antimicrobial peptides (Hollmann et al. 2018). Furthermore, the fact that modification through substitution of lipid A is plasmid encoded represents an important concern in the future of antimicrobial peptide drug development.

Acknowledgements This work was supported by Grants from Agencia Nacional de Promoción Científica y Tecnológica, Ministerio de Ciencia y Tecnología, Argentina, Fondo para la Investigación Científica y Tecnológica (PICT 2016-0478 and PICT 2017-2349), CONICET (PIP-2014-11220130100383CO), Universidad Nacional de Quilmes (PP-Microbiología básica y aplicada a agronomía, alimentos y salud), and Universidad Nacional de Santiago del Estero (PI-UNSE 23/A250). LS is a member of the Research Career of CIC-BA. DF, PCM and AH are members of the Carrera del Investigador Científico y Tecnológico (CONICET, Argentina). MM and PM acknowledge fellowships from CONICET.

\section{References}

Batzli KM, Love BJ (2015) Agitation of amyloid proteins to speed aggregation measured by ThT fluorescence: a call for standardization. Mater Sci Eng C 48:359-364. https://doi.org/10.1016/J. MSEC.2014.09.015

Bialvaei AZ, Samadi Kafil H (2015) Colistin, mechanisms and prevalence of resistance. Curr Med Res Opin 31:707-721. https://doi. org/10.1185/03007995.2015.1018989
Clinical and Laboratory Standards Institute C (2015) M02-A12: performance standards for antimicrobial disk susceptibility tests; approved standard-twelfth edition. Clin Lab Stand Inst 35:73

Deslouches B, Steckbeck JD, Craigo JK et al (2013) Rational design of engineered cationic antimicrobial peptides consisting exclusively of arginine and tryptophan, and their activity against multidrug-resistant pathogens. Antimicrob Agents Chemother 57:2511-2521. https://doi.org/10.1128/AAC.02218-12

Dong N, Zhu X, Chou S et al (2014) Antimicrobial potency and selectivity of simplified symmetric-end peptides. Biomaterials. https://doi.org/10.1016/j.biomaterials.2014.06.005

Esposito F, Fernandes MR, Lopes R et al (2017) Detection of colistin-resistant MCR-1-positive Escherichia coli using inhibition by EDTA and zeta potential assays. J Clin Microbiol JCM. https ://doi.org/10.1128/JCM.00835-17

Falagas ME, Kasiakou SK, Saravolatz LD (2005) Colistin: the revival of polymyxins for the management of multidrug-resistant gramnegative bacterial infections. Clin Infect Dis 40:1333-1341. https://doi.org/10.1086/429323

Hinchliffe P, Yang QE, Portal E et al (2017) Insights into the mechanistic basis of plasmid-mediated colistin resistance from crystal structures of the catalytic domain of MCR-1. Sci Rep. https:// doi.org/10.1038/srep39392

Hollmann A, Martinez M, Maturana P et al (2018) Antimicrobial peptides: interaction with model and biological membranes and synergism with chemical antibiotics. Front Chem. https://doi. org/10.3389/fchem.2018.00204

Li J, Nation RL, Turnidge JD et al (2006) Colistin: the re-emerging antibiotic for multidrug-resistant Gram-negative bacterial infections. Lancet Infect Dis 6:589-601. https://doi.org/10.1016/ S1473-3099(06)70580-1

Lim LM, Ly N, Anderson D et al (2010) Resurgence of colistin: a review of resistance, toxicity, pharmacodynamics, and dosing. Pharmacotherapy 30:1279-1291. https://doi.org/10.1592/ phco.30.12.1279

Liu Y-Y, Wang Y, Walsh TR et al (2016) Emergence of plasmidmediated colistin resistance mechanism MCR-1 in animals and human beings in China: a microbiological and molecular biological study. Lancet Infect Dis 16:161-168. https://doi. org/10.1016/S1473-3099(15)00424-7

Lowry GV, Hill RJ, Harper S et al (2016) Guidance to improve the scientific value of zeta-potential measurements in nanoEHS. Environ Sci Nano 3:953-965. https://doi.org/10.1039/c6en0 0136j

Magiorakos AP, Srinivasan A, Carey RB et al (2012) Multidrugresistant, extensively drug-resistant and pandrug-resistant bacteria: an international expert proposal for interim standard definitions for acquired resistance. Clin Microbiol Infect 18:268-281. https://doi.org/10.1111/j.1469-0691.2011.03570.x 
Poirel L, Jayol A, Nordmann P (2017) Polymyxins: antibacterial activity, susceptibility testing, and resistance mechanisms encoded by plasmids or chromosomes. Clin Microbiol Rev 30:557-596. https ://doi.org/10.1128/CMR.00064-16

Rapoport M, Faccone D, Pasteran F et al (2016) First description of mcr-1-mediated colistin resistance in human infections caused by Escherichia coli in Latin America. Antimicrob Agents Chemother 60:4412-4413. https://doi.org/10.1128/AAC.00573-16

Shah SR, Henslee AM, Spicer PP et al (2014) Effects of antibiotic physicochemical properties on their release kinetics from biodegradable polymer microparticles. Pharm Res 31:3379-3389. https ://doi.org/10.1007/s11095-014-1427-y

Turlej-Rogacka A, Xavier BB, Janssens L et al (2018) Evaluation of colistin stability in agar and comparison of four methods for MIC testing of colistin. Eur J Clin Microbiol Infect Dis 37:345-353. https://doi.org/10.1007/s10096-017-3140-3

Velkov T, Thompson PE, Nation RL, Li J (2010) Structure-activity relationships of polymyxin antibiotics. J Med Chem 53:18981916. https://doi.org/10.1021/jm900999h

Publisher's Note Springer Nature remains neutral with regard to jurisdictional claims in published maps and institutional affiliations. 